\title{
AT LEAST TWO FIFTHS OF THE ZEROS OF THE RIEMANN ZETA FUNCTION ARE ON THE CRITICAL LINE
}

\author{
J. B. CONREY
}

Let $\zeta(s)$ denote the Riemann zeta function defined for $s=\sigma+i t$ with $\sigma>1$ by

$$
\zeta(s)=\sum_{n=1}^{\infty} n^{-s} .
$$

Of central importance in number theory is the distribution of the complex zeros of $\zeta(s)$, all of which are in the critical strip $0<\sigma<1$ and are symmetrically located about the real axis and about the critical line $\sigma=$ $1 / 2$. Riemann conjectured in 1859 that all of these zeros are on the critical line; this conjecture, which is still unproved, is known as the Riemann Hypothesis.

The number of zeros of $\zeta(s)$ in the region $0<t \leq T$ of the critical strip is denoted $N(T)$ and is given asymptotically by

$$
N(T) \sim \frac{T}{2 \pi} \log T
$$

as $T \rightarrow \infty$. In 1942 Selberg [8] proved that a positive proportion of the zeros of $\zeta(s)$ are on the critical line; that is, if $N_{0}(T)$ denotes the number of zeros of $\zeta(s)$ in $0<t \leq T$ which are on the critical line, then Selberg's result is that

$$
\kappa=\liminf _{T \rightarrow \infty} \frac{N_{0}(T)}{N(T)}>0 .
$$

We will call $\kappa$ the proportion of zeros of $\zeta(s)$ on the critical line. (Note that $\kappa=1$ does not imply the Riemann Hypothesis.) Selberg did not give a numerical lower bound for $\kappa$.

In 1973 Levinson [7] developed a new method and proved that $\kappa>$ 1/3. It was noticed by Heath-Brown [5] and independently by Selberg that Levinson's method actually proves the stronger assertion that at least $1 / 3$ of the zeros of $\zeta(s)$ are simple and on the critical line.

We extend Levinson's method to prove

THEOREM 1. At least $2 / 5$ of the zeros of $\zeta(s)$ are simple and on the critical line.

Received by the editors April 25, 1988.

1980 Mathematics Subject Classification (1985 Revision). Primary 11M26; Secondary $11 \mathrm{~F} 37$.

Research supported by the Alfred P. Sloan Foundation, the NSF, and the Institute for Advanced Study. 
Levinson's method depends on the ability to give an asymptotic formula for the mean square on a vertical line segment $\{a+i t: T \leq t \leq 2 T\}$ of a linear combination of $\zeta(s)$ and its derivatives multiplied by a mollifier

$$
B(s)=\sum_{n \leq y} \frac{b(n)}{n^{s}} ;
$$

here $|1 / 2-a| \ll(\log T)^{-1}$ and the mollifier coefficients are given by

$$
b(n)=\mu(n) P\left(\frac{\log y / n}{\log y}\right)
$$

where $\mu$ is the Möbius function and $P$ is a polynomial which satisfies $P(0)=0$ and $P(1)=1$.

In Levinson's theorem, the length $y$ of the mollifier can be as large as $T^{1 / 2-\varepsilon}$ for any fixed $\varepsilon>0$ and the appropriate asymptotics can be deduced. The main new feature in Theorem 1 is that $y=T^{4 / 7-\varepsilon}$ is admissible for any $\varepsilon>0$. The work of Deshouillers and Iwaniec [4] on averages of Kloosterman sums plays the crucial role here. Their work is based in part on the Kuznetzov trace formula which relates sums of Kloosterman sums to Fourier coefficients of Maass cusp forms.

Our work also implies two density results for the zeros of $\zeta(s)$ which are strong near $\sigma=1 / 2$. Let $N(\sigma, T)$ denote the number of zeros $\rho=\beta+i \gamma$ of $\zeta(s)$ for which $\beta \geq \sigma$ and $0<t \leq T$.

THEOREM 2. For any $\varepsilon>0$ we have

$$
N(\sigma, T) \ll_{\varepsilon} T^{1-(8 / 7-\varepsilon)(\sigma-1 / 2)} \log T
$$

uniformly for $\sigma \geq 1 / 2$.

This theorem improves a result of Jutila [6].

THEOREM 3. We have

$$
\int_{1 / 2}^{1} N(\sigma, T) d \sigma \leq(0.0806+o(1)) T
$$

as $T \rightarrow \infty$.

This gives a small refinement of a result of Balasubramanian, Conrey, and Heath-Brown [1].

The details of the proof of Theorem 1 are in [3].

\section{REFERENCES}

1. R. Balasubramanian, J. B. Conrey, and D. R. Heath-Brown, Asymptotic mean square of the product of the Riemann zeta-function and a Dirichlet polynomial, J. Riene Angew. Math. 357 (1985), 161-181.

2. J. B. Conrey, Zeros of derivatives of Riemann's xi-function on the critical line, J. Number Theory 16 (1983), 49-74.

3. More than two-fifths of the zeros of Riemann's zeta-function are on the critical line, preprint.

4. J.-M. Deshouillers and H. Iwaniec, Kloosterman sums and Fourier coefficients of cusp forms, Invent. Math. 70 (1982), 219-288. 
5. D. R. Heath-Brown, Simple zeros of the Riemann zeta-function on the critical line, Bull. London Math. Soc. 11 (1979), 17-18.

6. M. Jutila, Zeros of the zeta-function near the critical line, Studies in Pure Mathematics, to the memory of Paul Turan, pp. 385-394 (Birkhäuser, Basel-Stuttgart, 1982).

7. N. Levinson, More than one-third of the zeros of Riemann's zeta-function are on $\sigma=$ 1/2, Adv. Math. 13 (1974), 383-436.

8. A. Selberg, On the zeros of Riemann's zeta-function, Skr. Norskevid. Akad. Oslo 10 (1942), 1-59.

9. E. C. Titchmarsh, The theory of the Riemann zeta-function, (2nd ed.) Clarendon Press, Oxford, 1986.

Department of Mathematics, Oklahoma State University, Stillwater, OklaHOMA 74078 
